\title{
Paroxysmal, exercise-induced, diurnally fluctuating dystonia: expanding the phenotype of SPG8
}

Eoin Mulroy ${ }^{1}$, Francesca Magrinelli1, 2, Nor Amelia Mohd Fauzi ${ }^{1}$, 3 , Shahedah Koya Kutty $^{1,4}$, Anna Latorre ${ }^{1}$, Kailash P Bhatia ${ }^{1}$

1 Department of Clinical and Movement Neurosciences, UCL Queen Square Institute of Neurology, London WC1N 3BG.

2 Department of Neurosciences, Biomedicine and Movement Sciences, University of Verona, Verona, Italy

3 Department of Medicine, Faculty of Medicine, Universiti Teknologi MARA Sungai, Buloh Campus, Selangor, Malaysia

4 Department of Internal Medicine, International Islamic University Malaysia, Pahang, Malaysia.

\section{Corresponding author:}

Eoin Mulroy

Department of Clinical and Movement Neurosciences, UCL Queen Square Institute of Neurology, London WC1N 3BG.

e-mail: e.mulroy@ucl.ac.uk

Funding: This research did not receive any specific grant from funding agencies in the public, commercial, or not-for-profit sectors.

Declarations of interest: none

Key words: Spastic Paraplegia, Hereditary; Parkinson Disease; Glut1 Deficiency Syndrome; Wiskott-Aldrich Syndrome Protein 


\section{Introduction}

The hereditary spastic paraplegias (HSP) are a heterogeneous group of neurodegenerative disorders characterized by length-dependent retrograde corticospinal tract degeneration, producing a clinical syndrome dominated by lower limb predominant spasticity. HSPs may display autosomal dominant, recessive, $\mathrm{X}$-linked or mitochondrial inheritance ${ }^{1}$. They are frequently categorized on clinical grounds into 'pure' HSPs - which can have additional bladder involvement and/or mild lower limb vibration sense abnormalities-and 'complex' HSPs, which comprise additional features such as neuropathy, seizures, cognitive decline, parkinsonism, dystonia, deafness and amyotrophy ${ }^{2}$.

SPG8 is a dominantly inherited, usually adult-onset HSP resulting from pathogenic variants in the WASHC5 gene. It has hitherto been known only to produce a pure HSP syndrome. Herein, we report paroxysmal exercise-induced dystonia(PED) as a novel phenotype of SPG8. We discuss important pitfalls on the road to diagnosis, suggest possible pathomechanisms underlying the development of PED in SPG8, and review less recognized causes of PED which are relevant to clinical practice.

\section{Case report}

A 56 years-old previously well female prolific marathon runner noted abnormal posturing of the left leg after running over $10 \mathrm{~km}$. Over time, symptoms worsened and would appear after progressively less distance. Aged 60 years, she noted similar symptoms in the right leg. Aged 62 years, she was evaluated in our movement disorder clinic, at which point symptoms were occurring upon walking. She denied lower limb sensory disturbance, or bowel/bladder symptoms. She noted improvement when walking backwards and could dance without problems. She described clear diurnal fluctuations, being markedly better in the morning. A trial of levodopa 300mg daily produced no benefit. She 
has one sister with 'gait difficulty', and a daughter with an 'unusual walk', but neither were available for review.

Examination 6 years following symptom onset showed bilateral abnormal leg posturing when walking forwards, which improved when walking backwards. Mirror movements upon finger tapping and gesture copying were evident. There was no parkinsonism. Lower limb tone, reflexes, proprioception and vibration sensation were normal. There was no clonus, but plantar responses were extensor. Upon further review 9 months later, she again demonstrated dystonia of both legs and trunk upon walking(see video).

Brain MRI and DATscan were normal. Cerebrospinal fluid glucose levels, pterins and monoamine metabolites were normal, and genetic testing for the TOR1A GAG deletion was negative. Central motor conduction times(CMCT) were normal. Because of the bilateral leg involvement and upgoing plantar responses, a nextgeneration sequencing gene panel for hereditary spastic paraplegias was requested which identified a heterozygous NM_014846.4:c.2505-1G>C p.(?) variant in the WASHC5 gene. This intronic mutation (CADD: 32; GERP: 2.35) has no entries in ClinVar and only one heterozygote in the apparently healthy population of gnomAD v3.1. It is predicted as disease causing by MutationTaster and suspected to alter the wild-type acceptor site (Genomnis HSF), most probably affecting normal splicing and leading to nonsense transcript of a gene in which loss-of-function has been described as mechanism of disease.

\section{Discussion}

Paroxysmal exercise-induced dystonia (PED) describes the occurrence of dystonia during exercise ${ }^{3}$. It carries a limited differential diagnosis, the most common aetiologies being Glut1 deficiency syndrome, young-onset Parkinson's disease and dopa-responsive dystonia. An approach to diagnosis, based on age of onset, structural and DAT neuroimaging and cerebrospinal fluid analysis has been proposed ${ }^{3}$. Though dystonia forms part of the phenotype in many complex 
HSPs, it is rarely an isolated presenting feature and PED has not, to our knowledge, been previously reported in any HSP.

Arriving at the correct diagnosis in this case was challenging not only because this phenotype is previously undescribed in SPG8, but also because of the presence of somewhat misleading 'pathognomonic' clinical features and diagnostic tests. For example, diurnal fluctuations (prominent in this case) are often considered pathognomonic of dopa-responsive dystonia (DRD) whereas in fact, they are described in other disorders including complex HSPs, SCA3 and early-onset Parkinson's disease ${ }^{4-8}$. Further, normal CMCTs should not discourage a diagnosis of HSP. Lower limb CMCTs may be normal in over $20 \%$ of people with HSP, despite severe spasticity on clinical examination, and have little correlation with disease severity 9 .

The pathophysiologic basis of PED in SPG8 is uncertain. The WASHC5 gene product, strumpellin, is a core component of the Wiskott-Aldrich syndrome protein and SCAR homolog (WASH) complex, which plays a major role in the sorting of certain surface proteins for either lysosomal degradation or recycling to the plasma membrane ${ }^{10}$. Interestingly, one of these proteins is Glut1. WASH complex dysfunction impairs Glut1 trafficking, and under conditions of stress, reduces cell surface Glut1 expression which may in some sense produce a 'Glut1 deficient state'11. It is also interesting to note that PED-associated young-onset Parkinson's disease may result from defective proteins that either directly (Vps35) or indirectly (Parkin) associate with this process ${ }^{12,13}$.

The differential diagnosis of PED continues to expand beyond the 'classic' causes(table 1) 3 $^{3}$ A thorough workup for rarer disorders (some of which are treatable), based on associated clinical features, neuroimaging, serum biochemistry, and genetic analysis is therefore paramount in cases with initial negative workup ${ }^{14}$. 
Acknowledgements: Nil

\section{Funding:}

Eoin Mulroy is supported by the Edmond J. Safra Foundation and the National Institute for Health Research University College London Hospitals Biomedical Research Centre. Francesca Magrinelli is supported by the European Academy of Neurology (EAN) Research Fellowship 2020. Kailash P Bhatia holds research grants from EU Horizon 2020 and has received honoraria to speak at meetings or to attend advisory boards from Ipsen, Cavion, Allergan, Teva Lundbeck and Bial pharmaceutical companies. He also receives royalties from Oxford University Press and a stipend for MDCP editorship. Nor Amelia Mohd Fauzi, Shahedah Koya Kutty and Anna Latorre report no disclosures.

\section{Ethics statement:}

All procedures followed were in line with the journal's ethics policy and the subject gave consent to be videoed for publication both in print and online.

\section{Authors' contributions:}

$1 \mathrm{~A}$, conception and design of the study; $1 \mathrm{~B}$ : acquisition of data; $1 \mathrm{C}$, analysis and interpretation of data.

$2 \mathrm{~A}$, drafting the article; $2 \mathrm{~B}$, revising it critically for important intellectual content 3 , Final approval of the version to be submitted. 
SKK

1C, 2A, 2B, 3

$\mathrm{AL}$

$1 \mathrm{C}, 2 \mathrm{~A}, 2 \mathrm{~B}, 3$

KPB

$1 \mathrm{C}, 2 \mathrm{~A}, 2 \mathrm{~B}, 3$

\section{References}

1. Finsterer J, Löscher W, Quasthoff S, et al. Hereditary spastic paraplegias with autosomal dominant, recessive, X-linked, or maternal trait of inheritance. J. Neurol. Sci. 2012;318(1-2):1-18.

2. Harding AE. Hereditary 'pure' spastic paraplegia: a clinical and genetic study of 22 families. J. Neurol. Neurosurg. Psychiatry 1981;44(10):871883.

3. Erro R, Stamelou M, Ganos C, et al. The Clinical Syndrome of Paroxysmal Exercise-Induced Dystonia: Diagnostic Outcomes and an Algorithm. Mov. Disord. Clin. Pract. 2014;1(1):57-61.

4. Yamamura Y, Hattori N, Matsumine H, et al. Autosomal recessive earlyonset parkinsonism with diurnal fluctuation: clinicopathologic characteristics and molecular genetic identification. Brain Dev. 2000;22:87-91.

5. Rohé CF, Montagna P, Breedveld G, et al. Homozygous PINK1 C-terminus mutation causing early-onset parkinsonism. Ann. Neurol. 2004;56(3):427431.

6. Wilder-Smith E, Tan E., Law H., et al. Spinocerebellar ataxia type 3 presenting as an l-DOPA responsive dystonia phenotype in a Chinese family. J. Neurol. Sci. 2003;213(1-2):25-28.

7. Doherty KM, Silveira-Moriyama L, Parkkinen L, et al. Parkin Disease. JAMA Neurol. 2013;70(5):571.

8. Wijemanne S, Shulman JM, Jimenez-Shahed J, et al. SPG11 Mutations Associated With a Complex Phenotype Resembling Dopa-Responsive Dystonia. Mov. Disord. Clin. Pract. 2015;2(2):149-154.

9. Siow S-F, Cameron Smail R, Ng K, et al. Motor Evoked Potentials in 
Hereditary Spastic Paraplegia-A Systematic Review. Front. Neurol. $2019 ; 10$

10. Steinberg F, Gallon M, Winfield M, et al. A global analysis of SNX27retromer assembly and cargo specificity reveals a function in glucose and metal ion transport. Nat. Cell Biol. 2013;15(5):461-471.

11. Piotrowski JT, Gomez TS, Schoon RA, et al. WASH Knockout T Cells Demonstrate Defective Receptor Trafficking, Proliferation, and Effector Function. Mol. Cell. Biol. 2013;33(5):958-973.

12. Williams ET, Glauser L, Tsika E, et al. Parkin mediates the ubiquitination of VPS35 and modulates retromer-dependent endosomal sorting. Hum. Mol. Genet. 2018;27(18):3189-3205.

13. Williams ET, Chen X, Moore DJ. VPS35, the Retromer Complex and Parkinson's Disease. J. Parkinsons. Dis. 2017;7(2):219-233.

14. Garone G, Capuano A, Travaglini L, et al. Clinical and Genetic Overview of Paroxysmal Movement Disorders and Episodic Ataxias. Int. J. Mol. Sci. 2020;21(10):3603. 